\title{
A predictive formula of the contraction stress in restorative and luting materials attending to free and adhered surfaces, volume and deformation
}

\author{
A. Miguel, J.C. de la Macorra* \\ Conservative Dentistry Department, Faculty of Odontology, Complutense University, Madrid, Spain
}

\begin{abstract}
Objectives: To find a predictive formula of stress, considering the surfaces (free, adhered) involved, the volume and characteristics of material and the deformation of the measuring system.

Materials and methods: 231 samples of five chemically cured restoratives (Silar (SIL, 23), Clearfil F2 (CLE, 39), P10 (P10, 33), Concise (CON, 30), Isopast (ISO, 28)) and four luting (3M Experimental 241 (EXM, 20), Variolink II (VAR, 13), Vitremer LC (VTM, 20) and Dyract Cem (DYR, 25)) materials were allowed to polymerize until they reached a maximum tension $\left(T_{\max }, 25\right.$ min) between six pairs $(\varnothing 5.81,8.5$, $11.26,12.42,17.02,23.14 \mathrm{~mm}$ ) of polished metallic discs (range of distances: 0.02-5.9 mm) mounted in a tension machine. The deformation of the measuring system was measured for the recorded forces.

Results: A descriptive non-linear formula $T_{\max }=K \mathrm{Vol}^{-3.267} \mathrm{FS}^{3.283} \mathrm{AS}^{0.642} \mathrm{Def}^{0.561}$ was found that individualizes the material's characteristics $(K)$ that considers volume (Vol), free (FS) and adhered (AS) surfaces and deformation (Def) of the system for each force. This formula renders good correlation (material $K$ ( $r^{2}$ coefficient)): SIL 0.9998 (0.995), CLE 1.0062 (0.989), P10 1.0224 (0.990), CON 0.9908 (0.992), ISO 0.9648 (0.974), EXM 1.0083 (0.991), VAR 0.9777 (0.996), VTM 0.9925 (0.993), DYR $0.9971(0.997)$ between actual $T_{\max }$ and calculated Tension. There are statistically significant differences $(p=0.002)$ between $K$ values of both (restorative and luting) groups.

Significance: Predictive parameters have influence in a different way to what is actually considered, if the system is allowed to have deformation, as occurs naturally and volume and material's characteristics are considered. (C) 2001 Academy of Dental Materials. Published by Elsevier Science Ltd. All rights reserved.
\end{abstract}

Keywords: Polymerization; Stress; Configuration

\section{Introduction}

Composite resin materials are widely used in Odontology not only for filling cavities, but also for reconstructing dental structures, luting restorations and orthodontic appliances. The main problems posed by these materials are marginal failure and recurrent caries, originating in most cases, because of the polymerization contraction, which competes with adhesion to the dental tissues [1,2].

It has been calculated in vitro that these materials have an approximate volumetric loss of $1.35-4.9 \%$ due to polymerization contraction [3-5]. This contraction, when the material is in contact with some surface or inside a cavity, is limited by the adhesion to the surrounding walls, generating stress at the material, the interface and/or the structures at which it is adhered (tooth or cemented restoration) [6-8].

The generation of these tensions varies as polymerization progresses [9]. At the beginning, the resin molecules can slide over each other. Thus, the material mass changes its shape to adapt to the new volumetric situation created by the contraction, without damage to its structure or interfaces. This deformation is known as flow. As curing progresses, the rigidity of the material increases, resulting in flow decrease and the buildup of stress [1]. This stress, if it reaches a certain level, can result in a defect of the interface with possible consequences: microfiltration of fluids and recurrent caries, the impairment of the mechanical properties or cohesive failure in the material, post-operative sensitivity and/or fracture of the tooth or the cemented restoration.

The polymerization contraction behavior and the flow capacity of a material depend on its physical and chemical characteristics [10], environmental conditions (temperature, humidity...) and on the cavity design. 
Table 1

Materials used

\begin{tabular}{llll}
\hline Material & Manufacturer & Type & Batch \\
\hline CLEARFIL F2 & CLE & KURARAY & Composite restorative resins \\
ISOPAST & ISO & VIVADENT & Composite restorative resins \\
SILAR & SIL & $3 \mathrm{M}$ & Composite restorative resins \\
P10 & P10 & $3 \mathrm{M}$ & Composite restorative resins \\
CONCISE & CON & $3 \mathrm{M}$ & Composite restorative resins \\
EXM241 & EXM & $3 \mathrm{M}$ & Composite resin cements \\
VARIOLINK II & VAR & VIVADENT & Composite resin cements \\
VITREMER LC & VTM & $3 \mathrm{M}$ & Resin modified glass ionomer cement \\
DYRACT CEM & DYR & DeTrey & Resin modified glass ionomer cement \\
\hline
\end{tabular}

In 1987, Feilzer and colleagues [11] published a study, already a classic, in which they studied in vitro the tensional behavior of adhesive materials in different simulated cavity situations. Their experimental design involved application of the material between two disks attached to a tensiontraction machine, recording the tensions generated during polymerization. Throughout the process, and with the aim of creating a rigid system, they established a mechanism that detected the changes in distance between the discs and forced the recovery of initial distance. In this way they studied different cylindrical cavities, which they defined by the configuration factor $(C)$ determined as the ratio of adhered to the free surfaces:

$C=$ adhered surface/free surface

They concluded that the configuration factor predicts the tensional behavior of the adhesive materials placed in a cavity so that for the same material with a lower $C$, increased flow will be allowed and therefore less stress on the material will occur on the interface, the tooth and/or the restoration.

This study has been widely used as a reference since its publication because it meant a crucial advance in the understanding of tensional behavior of materials placed in a cavity. However, in our opinion, it seems to have a series of limitations due to its very conception and the supposedly rigid situation in which the material is placed.

The first limitation is that there is no rigidity in the system, since the discs are continually coming closer because of the tensions produced by the setting material and separating because of the distance recovery system. This constant back and forth movement may induce errors in the readings since we may suppose that the material is partially stressed.

The second limitation is conceptual: no pure rigid system exists in nature and especially not in the sense previously explained. The tooth and the restoration inevitably possess a certain deformation capacity, which allows partial stress relaxation of the polymerization tensions produced.

The third is that it does not consider other important factors, namely the volume of the restoration and the specific material characteristics.

Finally, the configuration factor, as defined, is the trans- lation of a general concept that does not permit numerical approximation of the problem.

Therefore, the purpose of this paper is to study the tensions, which are generated during the polymerization of different composite resin materials in an environment with known deformation, to determine if there are different tensional behaviors among materials and to try to define the numerical relationship of different factors with tensional behavior, applicable to elastic systems.

\section{Materials and methods}

We studied nine chemical cured materials, five composite resin restoratives: Silar, P10 and Concise (3M Co, St Paul, MN, USA), Clearfil F2 (Kuraray, Osaka, Japan) and Isopast (Ivoclar/Vivadent, Schaan, Liechtenstein)), two composite resin cements: experimental resin EXM 241 (3M), Variolink II (Ivoclar/Vivadent)) and two resin modified glass ionomer cements: Vitremer LC (3M) and Dyract Cem (DeTrey/Dentsply GmbH, Konstanz, Germany) (Table 1),

Samples of these materials were placed in a pressuretraction machine (Hounsfield 5000M, England) as follows:

- Each restorative material was mixed and placed between a pair of metallic discs of six different diameters $(5.81,8.5,11.26,12.42,17.02$ and $23.14 \mathrm{~mm}$ ). The surface of each disc was polished with a 120-grit paper (Struers, Denmark) and separated by distances ranging between 0.02 and $5.9 \mathrm{~mm}$.

- For luting materials, only $23.14 \mathrm{~mm}$ diameter discs were used. Mixed material was placed between the discs and these were approximated until a centered disc of material was obtained. Once the test was completed the sample area was measured with an image analyzer (Leica Q WIN 500).

All samples were allowed to polymerize for $25 \mathrm{~min}$ recording the force (maximum speed of readings: 20 readings/s). During this time the lower, movable, arm of the pressure-traction machine was in a stationary position, and the room temperature was registered. Time elapsed 


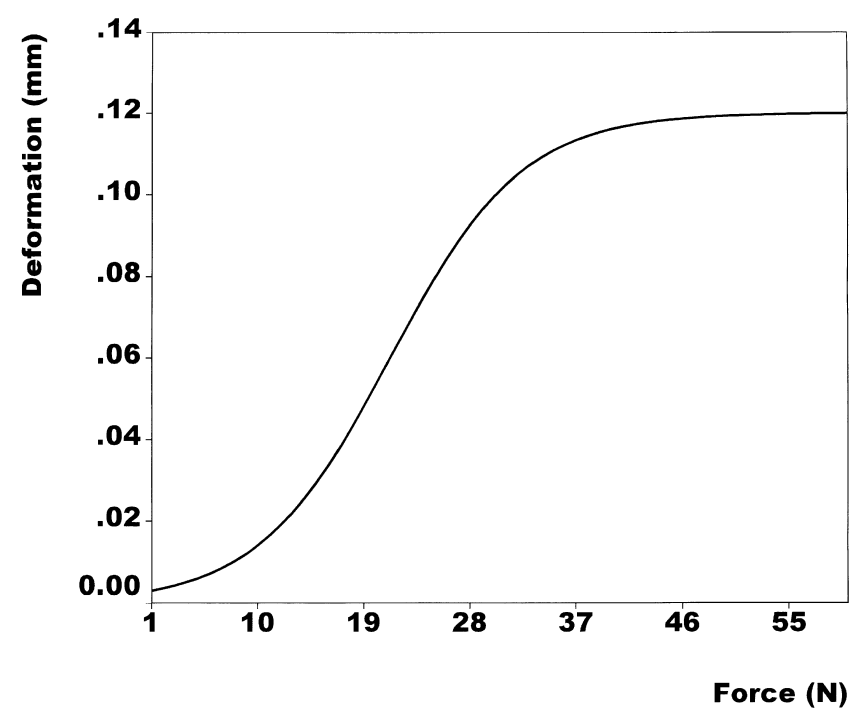

Fig. 1.

between the start of mixing and the beginning of the experiment was also recorded.

Since the system is not rigid, in order to determine the deformation of the pressure-traction machine and the connection mechanisms for each force level, we carried out 10 traction tests with the sample holders fixed one to another. Using the force readings and the resulting deformation, we obtained a graphic representation (Fig. 1) of the non-linear equation, which described the deformation as a function of force. In this way, it was possible to determine (calculate) the system deformations in each case according to the recorded force.

The maximum tension produced $\left(T_{\max }\right)$ was calculated by dividing the maximum force $\left(F_{\max }\right)$ by the surface area upon which the force was exerted (upper disc in the restoratives, and upper material area in the luting materials).

Grouping together all samples, we looked (SPSS ver 9, SPSS INC, Chicago, IL) for the coefficients $A, B, C$ and $D$ that best fit the non-lineal formula

$$
T_{\max }=\operatorname{Vol}^{A} \mathrm{FS}^{B} \mathrm{AS}^{C} \operatorname{Def}^{D}
$$

where Vol is the volume of the sample $\left(\mathrm{mm}^{3}\right)$, FS the free surface $\left(\mathrm{mm}^{2}\right)$, AS the total adhered surface $\left(\mathrm{mm}^{2}\right)$ and Def the deformation of the system for $T_{\max }(\mathrm{mm})$.

In order to individualize each material, with the coefficients $A, B, C$ and $D$ found, a constant value, $K$, was calculated for each material with the formula

$K=T_{\max } / \operatorname{Vol}^{A} \mathrm{FS}^{B} \mathrm{AS}^{C} \operatorname{Def}^{D}$

$K$ means were compared for the two (restorative and luting) groups of materials with Kolmogorov-Smirnov non-parametric test because their distributions were far from normal $(K-S$ test for normality significance $p<0.000029)$ and
Table 2

$K$ constants for each material and predictive power

\begin{tabular}{llrll}
\hline Material & $m^{\mathrm{a}}$ & \multicolumn{1}{c}{$n^{\mathrm{b}}$} & $\sigma^{\mathrm{c}}$ & $r^{2 \mathrm{~d}}$ \\
\hline SIL & 0.9998 & 23 & 0.670 & 0.995 \\
CLE & 1.0062 & 39 & 0.105 & 0.989 \\
P10 & 1.0224 & $30(3)$ & 0.104 & 0.990 \\
CON & 0.9908 & 30 & 0.093 & 0.992 \\
ISO & 0.9648 & 28 & 0.064 & 0.974 \\
EXM & 1.0083 & 20 & 0.056 & 0.991 \\
VAR & 0.9777 & 13 & 0.020 & 0.996 \\
VTM & 0.9925 & $18(2)$ & 0.037 & 0.993 \\
DYR & 0.9971 & 25 & 0.006 & 0.997 \\
\hline
\end{tabular}

a $m$ : mean values.

b $n$ : number of samples (number of detached samples).

c $\sigma:$ standard deviation.

d $r^{2}$ : correlation coefficient between actual $T_{\max }$ and predicted tension.

their variance differed significantly (Levene test for homocedasticity significance, $p<0.009$ for both groups).

To check the behavior of $C$ factor in an elastic system, we plotted $C$ factor versus $T_{\max }$.

\section{Results}

The deformation suffered by our system for any recorded force in the range $0-60 \mathrm{~N}$ follows the equation:

Def $=\frac{0.12}{1+\left[45.48 \mathrm{e}^{-0.18 F}\right]}$,

where Def is the deformation in $\mathrm{mm}$ and $F$ the force in $\mathrm{N}$ (Fig. 1).

The general formula with the estimated coefficients, with all the materials grouped, predicting the maximum tension is: $T_{\max }=\mathrm{Vol}^{-3.267} \mathrm{FS}^{3.283} \mathrm{AS}^{0.642} \operatorname{Def}^{0.561}$. This formula has an $r^{2}=99.17 \%$.

$K$ constant values obtained per material from the formula $K=T_{\max } / \mathrm{Vol}^{-3.267} \mathrm{FS}^{3.283} \mathrm{AS}^{0.642} \mathrm{Def}^{0.561}$ are listed in Table 2. In total, five samples were discarded because of detaching before total time was elapsed.

$K-S$ non-parametric test comparing $K$ values of both groups' (luting and restorative) results were that they vary significatively $(Z=1.849, p=0.002)$.

Correlation was found between actual $T_{\max }$ and predicted tension (by the formula), for each individual material (Table 2). Fig. 2 shows relationship between $C$ factor and $T_{\max }$.

\section{Discussion}

Until now, the only way to anticipate the tensional behavior of adhesive materials was by means of the configuration factor [11], defined as

$C=$ adhered surface/free surface 


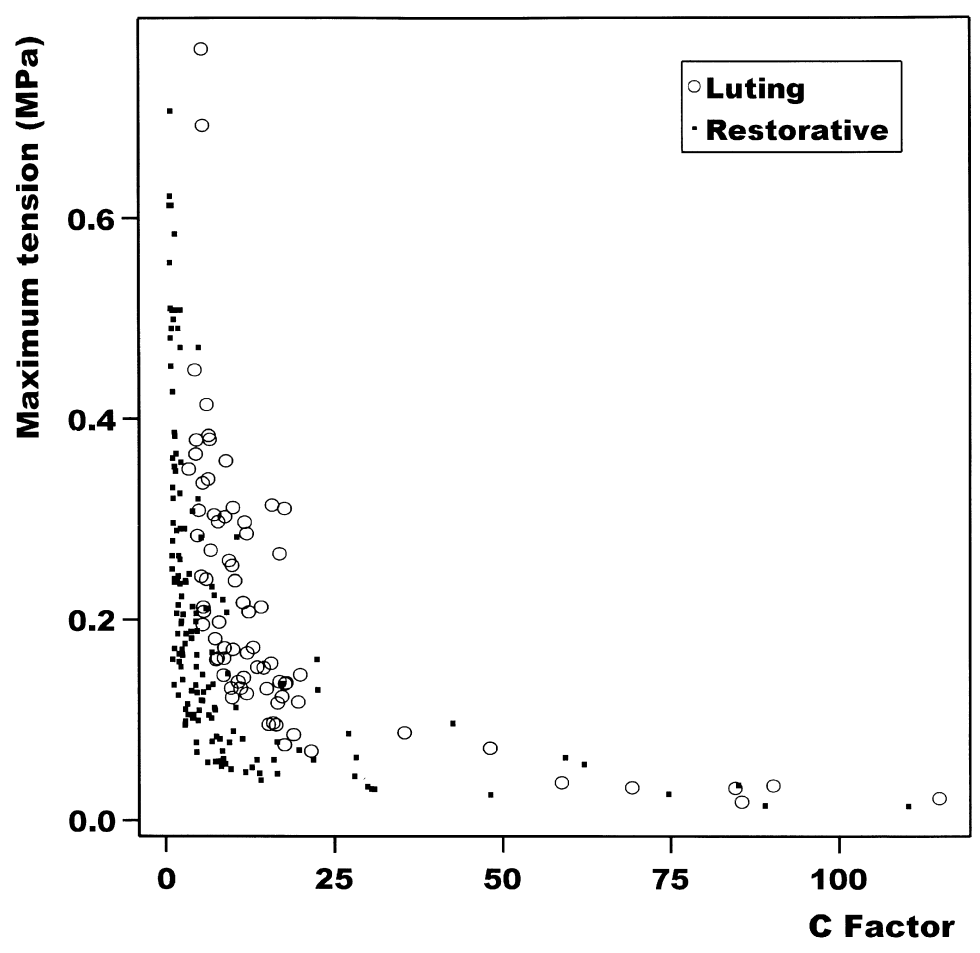

Fig. 2.

In our opinion, although this formulation had a high conceptual value at the time of its publication, it should be revised since it cannot be individualized for each material and does not offer a precise predictive value. Moreover, as shown in Fig. 2, $C$ factor has, in a deformable setup, an unexpected relationship with maximum tension. This relationship is not linear (as suggested by the intrinsic formulation of $C$ factor: higher $C$, higher tension) and, more importantly, association is inversed: higher $C$ factors produce lower tensions.

We believe that this is due to the fact that although the $C$ factor considers the configuration of the cavity, it ignores important parameters such as the deformation of the adhesive walls or the specific characteristics of each material and the volume of the restorative undergoing polymerization and to the fact that it was formulated in a rigid setup. Considering all these, we have been able to define a relationship in the way that maximum tension can be predicted very precisely. This type of statistical approach leads to a predictive formula of tension pertaining to other parameters, with an apparent inconsistency in units, in the same way that a relationship could be found, for instance, between the speed $(\mathrm{m} / \mathrm{s})$, weight $(\mathrm{kg})$ and horsepower $(\mathrm{CV})$ of an automobile, and obtain a valuable expression of a mathematical relationship (for instance, speed $=\mathrm{HP}^{\mathrm{A}} \cdot$ weight $^{\mathrm{B}}$ ).

\subsection{System compliance}

It has always been thought that the studies on contraction stress should be carried out in rigid systems in order to be able to record all the tensions generated during polymeriza- tion. It was believed that if the system is deformable, its elasticity or compliance would hinder the obtaining of realistic stress data since an unknown part of the tensions generated by the material would relax due to the deformation of the system [2]. Moreover, these assumed errors would not affect all cases in the same way due to the fact that this elasticity is relatively constant. So, smaller samples would permit the relaxation of a larger percentage of the stress (resulting in much lower stress data), while in larger samples the reading would only be slightly less [12].

This view has lead to the development of supposedly rigid systems on the one hand, and on the other hand, to the skepticism about the results of works that did not use "rigid" experimental setups.

The problem of designing a rigid environment to study the tensions generated by materials during polymerization is that no purely rigid system exists for carrying out the necessary measurements. All known systems are based on the use of a load cell, which is only able to perceive the forces through its deformation. Moreover, all the systems require connection mechanisms between the cell and the material being studied, which also have a certain degree of elasticity.

As we mentioned before, the most accepted solution that has been proposed is that of Feilzer and colleagues [11]. It proposes a feedback mechanism, which detects changes in the initial distance between the discs and corrects them. This method has two disadvantages that impair its effectiveness. In the first place, since it continues to permit slight deformations, it does not create a truly rigid environment for the material. In the second place the constant back and forth movements of the movable disc is most likely to cause 
distortions in the readings since they stress the material by subjecting it to external traction after allowing minimum contraction.

We focus the problem in the following way: we know that our experimental system (the load cell and the connection mechanisms) can be deformed, as is the case for any measuring system. What we really want to know when we study the tensions generated by a material during polymerization contraction is: what tensions will this material exert on the dental tissue or on the restoration material? And, are these tensions going to endanger the tooth-restoration interface and/or its internal cohesion?

The tooth-restoration complex and our measuring system can be deformed, albeit not to the same extent. This deformation will always prevent the appearance of part of the tension that would be generated by the material in a hypothetical purely rigid environment. The tensions that are not compensated for by the deformation, which in our experiment would be recorded by the load cell, are those that can reach (and possibly alter) the interface and/or generate stress in the tooth or the restoration, and are consequently of most interest to us. With regard to the study of contraction stress, the main difference between our system and any other non-rigid system with the tooth-restoration complex is that the elasticity is different. If we consider the deformation results, the studies carried out with non-rigid systems are valid. Moreover, they are the only ones that let us come as close as possible to what really occurs during the polymerization tension of materials.

Compared to other authors [10,11], our records of tension are low. The reason is as we have just explained: the deformation of the system. It is probable that the dentin is even more deformable than the pressure-traction mechanisms of the machine that we use. The dentin is subjected to traction Young's modulus is $13.7 \pm 3.4 \mathrm{GPa}[13]$ and studied with indentation between 10.3 and $29.8 \mathrm{GPa}[14,15,16]$. Stainless steel's module is approximately $200 \mathrm{GPa}$ [17], which suggests that in reality the clinical tension figures may be even smaller.

In our formulation, $T_{\max }$ is directly proportional to Def raised to the power of 0.561 . This is apparently in opposition to what we have just said: it suggests that more deformation will cause more tension. We must have in mind that we calculate the deformation, not the modulus. In our experimental setup, higher $T_{\max }$ recording corresponded with higher deformations, as Def was calculated from the maximum force registered, so they must be directly correlated. To apply this formulation to other systems, their deformation/force relation should be first measured in a similar way.

\subsection{Cavity configuration}

The cavity characteristics have been thoroughly studied by Feilzer and colleagues [11]. They conclude that the geometric distribution of the cavity could be defined by the quotient between the adhered surface (AS) and the free surface (FS) and that this quotient, called the configuration factor or the $C$ factor, makes it possible to predict the tension behavior of the adhesive material. The higher the factor, the greater the tensions produced on the adhesive surfaces, in their experimental design. Using Silar and P10, for $C=1$ values the stress relaxation caused by flow is sufficient to maintain the consistency of the samples and the interface. When $1<C<2$, the results are inconsistent and predict that the adhesives at that time currently available would not produce dependable results. For $C>2$, all the samples broke cohesively.

According to our data, the cavity defined by the adhered surface and the free surface affects the magnitude of the tensions, but it is not the only factor responsible for the tension. Moreover, our results suggest that the influence of the free surface and the adhered surface is not the same; the former has a greater effect than the latter. Also, we have found that the volume of the sample has an important role in the prediction of the final tension.

Volume of "real" cavities can be calculated [18], resulting in mean total volume of restoration for class I cavities of $21.3 \mathrm{~mm}^{3}(n=10, \mathrm{sd}=8.9)$ and for class II cavities of $65.1 \mathrm{~mm}^{3}(n=5, \mathrm{sd}=12.9)$.

Our approach proposes a conceptual change with regard to the prior formulation proposed by Feilzer and colleagues in 1987 [11], since the "classic" configuration factor formula is a conceptual one, and ours is a numerical approach. In this way, it is difficult to evaluate each geometrical factor alone, as they are, of course, mathematically interrelated.

As is to be expected, the stresses that occur during the polymerization of a composite resin are not unidirectional [19]. There must be components of this stress parallel to the adhesive interfaces that cause shearing stresses that are different in degree and direction according to the zone being studied. This is due to the material's need to move into the available space in order to adapt to the new volumetric situation, not just by deforming the free surface. This means that for larger adhesive areas, the movements of the material's mass - and consequently the stresses parallel to the adhered surface(s) — will be greater and the relation FS/ AS/Vol will change also.

Another probable consequence of this phenomenon is that the translation of a cylindrical cavity (such as that used by us), with parallel adhesive surfaces situated at the bases of a cylinder, to a real cavity (more complex), is mistaken. In our opinion, the geometry of real cavities, with irregular nonparallel walls, obliges to a much more complex spatial distribution of normal and oblique stresses on the walls than the ones which we are able to currently record.

In our experimental design, samples were stuck to the discs just by mechanical interlock-discs were polished before each measurement. This type of adhesion may allow some displacements parallel of the composite resin to the adhesive interface, relaxing some shear stress. This may not happen in real cavities without 
detrimental consequences to the final sealing of the interface.

\subsection{Characteristics of the material}

With regard to the characteristics of the material, it can be assumed that since the chemical (such as the type and degree of polymerization) and physical (viscosity or volumetric contraction percentage during polymerization) properties of the materials vary, the tensional behavior will be different even though the cavity and compliance characteristics were the same. In the materials we have studied, we have found statistically significant $(p=0.002)$ differences among $\mathrm{K}$ values, between the restorative materials group (SIL, CLE, P10, CON and ISO) and that of luting materials (EXM, VAR, VTM and DYR).

This is an important find, since it has to be remembered that $K$ is forced to convex around the value of 1 , because it is obtained by a simple division in a general predictive formula with a high correlation coefficient $\left(r^{2}=0.9917\right)$.

In this way, the fact that there are statistically significant differences between $K$ values in the two groups of materials means, in our opinion, that material characteristics have to be considered in prediction of the tension such material will yield when polymerizing. Table 2 shows predictive power $\left(r^{2}\right)$ of the formula per material, assuming each individual $K$ coefficient.

For us, this differential $K$ factor must contain more information than we are currently able to distinguish about the material (viscoelastic characteristics, static and dynamic modules of elasticity, effectiveness of the reaction, type, amount and properties of filling, porosity, etc.) and the environmental conditions (temperature, humidity, pressure, etc.).

\section{Acknowledgements}

This study was performed as partial fulfillment of the $\mathrm{PhD}$ degree requirements (Dr Miguel) and was funded, in part, by a predoctoral grant (Dr Miguel) BE350/95-AP95 of the Ministerio de Educación y Ciencia, Spain. Authors want to acknowledge all manufacturers for kindly providing the materials studied, Ivoclar/Vivadent representatives in Spain, for aiding in the development of software used, and Dr G. Eliades for help in editing the manuscript.

\section{References}

[1] Feilzer AJ, de Gee AJ, Davidson CL. Quantitative determination of stress reduction by flow in composite restorations. Dent Mater 1990;6:167-71.

[2] Bouschlicher MR, Vargas MA, Boyer DB. Effect of composite type, light intensity, configuration factor and laser polymerization on polymerization contraction forces. Am J Dent 1997;10:88-96.

[3] Puckett AD, Smith R. Method to measure the polymerization shrinkage of light-cured composites. J Prost Dent 1992;68:56-58.

[4] Lai JH, Johnson AE. Measuring polymerization shrinkage of photoactivated restorative materials by a water-filled dilatometer. Dent Mater 1993;9:139-43.

[5] Tolidis K, Nobecourt A, Randall RC. Effect of a resin-modified glass ionomer liner on volumetric polymerization shrinkage of various composites. Dent Mater 1998;14:417-23.

[6] Davidson CL, de Gee AJ, Feilzer AJ. The competition between the composite-dentin bond strength and the polymerization contraction stress. J Dent Res 1984;63(12):1396-9.

[7] Davidson CL, Van Zeghbroeck L, Feilzer AJ. Destructive stresses in adhesive luting cements. J Dent Res 1991;70(5):880-2.

[8] Meredith N, Setchell DJ. In vitro measurement of cuspal strain and displacement in composite restored teeth. J Dent 1997;25:331-7.

[9] Watts DC, Cash AJ. Determination of polymerization shrinkage kinetics in visible-light-cured material: methods development. Dent Mater 1991;7:281-7.

[10] Condon JR, Ferracane JL. Polymerization contraction stress of commercial composites. J Dent Res 1998;77:639.

[11] Feilzer AJ, de Gee AJ, Davidson CL. Setting stress in composite resin in relation to configuration of the restoration. $J$ Dent Res 1987;66:1636-9.

[12] Davidson CL, Feilzer AJ. Polymerization shrinkage and polymerization shrinkage stress in polymer-based restoratives. $J$ Dent 1997;25:435-40.

[13] Sano H, Takatsu T, Ciucchi B, Rusell CH, Pasley DH. Tensile properties of resin-infiltrated demineralized human dentin. J Dent Res 1995;74(4):1093-102.

[14] Xu HHK, Smith DT, Jananmir S, Romberg E, Kelly JR, Thompson VP, Rekow ED. Indentation damage and mechanical properties of human enamel and dentin. J Dent Res 1998;77(3):472-80.

[15] Kinney JH, Balooch M, Marshall SJ, Marshall JR, Weihs TP. Hardness and Young's modulus of human peritubular and intertubular dentine. Arch Oral Biol 1996;41(1):9-13.

[16] Meredith N, Sherriff M, Setchell DJ, Swanson SAV. Measurement of the microhardness and Young's modulus of human enamel and dentine using an indentation technique. Arch Oral Biol 1996;41(6):539-45.

[17] Alster D. Dental resin joints. Stress and strength. PhD thesis, Amsterdam, 1996.

[18] Macorra JC, Gomez-Fernandez S. Quantification of configuration factor in Class I and II cavities and simulated cervical erosions. Eur J Prost Res Dent 1996;4(1):29-33.

[19] Kinomoto Y, Torii M. Photoelastic analysis of polymerization contraction stress in resin composite restoration. $\mathrm{J}$ Dent 1998;26:165-71. 